Gabriel Maxime DONG MOUGNOL* Willy Didié FOGA KONEFON**

\title{
MIGRATIONS ET COHABITATION POLITIQUE INTERETHNIQUE DANS UN QUARTIER DE DOUALA AU CAMEROUN : LE CAS DE NEW-BELL, 1913-1990
}

\author{
Migration and Political Interethnic Cohabitation in a Neighbourhood of Douala, \\ Cameroon. The case of New-Bell, 1913-1990
}

Abstract: The New-Bell Neighbourhood was established by the German colonial authorities with a view to settle the dual community of the Bell clan, which had been expropriated of their land possessions. This area gradually turned into a safe haven for various populations that immigrated to Douala.

The aim of this paper is to highlight the ways in which various New-Bell communities got involved in the local and national political life throughout the period that spans from 1913 to 1990. The research is based on sociological inquiries, oral history investigations, archival documents and books dedicated to the political life in Cameroon.

Keywords: Cameroon, Douala, New-Bell, immigration, ethnic community

\section{Introduction}

L'une des spécificités du Cameroun est sa grande richesse ethnique. Ce pays d'Afrique centrale qui compte environ trois cents ethnies, porte fièrement son qualificatif d' «exception plurielle » dans le continent. ${ }^{1}$ Bien que cette exception soit source d'une certaine richesse pour le pays, il convient cependant d'être en accord avec Charly Gabriel Mbock qui pense que « s'il est courant d'entendre dire que la configuration sociologique du Cameroun est un atout, grâce à sa diversité ethnique et culturelle, ce discours politique n'est pas toujours suivi d'action politique conséquent $»^{2}$.

\footnotetext{
* Université de Yaoundé, Cameroun (dongmougnol2000@yahoo.fr)

** Université de Yaoundé I, Cameroun (willydidie@yahoo.fr)

${ }^{1}$ Pour plus de détails, lire KegneFodouop, Le Cameroun Autopsie d'une exception plurielle en Afrique, Paris, L'Harmattan, 2010.

${ }^{2}$ C. G. Mbock (ss la dir. de), Les conflits ethniques au Cameroun : quelles sources, quelles solutions?, Yaoundé, Edition Saagraph, 2000, p. 9.
}

Analele Universităţii „Dunărea de Jos” din Galaţi, Seria 19, Istorie, tom XVII, 2018, pp. 157182. 
New-Bell, quartier populeux de Douala ${ }^{3}$, s'illustre comme un exemple de cohabitation pluriethnique à caractère nationale et internationale. La gestion de cette diversité des populations est faite d'une alternance fréquente de stabilités et d'instabilités dans la cohabitation interethnique. Ceci au rythme de l'évolution de l'actualité politique nationale.

Ce bouillonnement politico-ethnique amène le chercheur à s'interroger premièrement sur les motifs de création de ce quartier et l'origine des populations qui l'habitent. Il serait par la suite intéressant de comprendre le processus qui a défini leur installation. Quelle a souvent été l'attitude adoptée par chaque groupe ethnique face aux grands événements politiques qu'a eu à traverser le Cameroun?

Cette problématique s'insère dans un cadre chronologique bien précis qui va de 1913, date probable de la naissance de New-Bell, à1990, année de la réinstauration du multipartisme au Cameroun. Pour mieux appréhender les faits liés à cette problématique, nous optons pour une analyse en deux étapes. La première est essentiellement basée sur les migrations et installations des grands groupes ethniques actuels de New-Bell, puis la seconde s'intéresse aux manifestations de la cohabitation politique au sein de ces peuples.

\section{I - De la naissance de New-Bell aux migrations des populations allogènes A- Expropriation foncière des Duala et naissance de New- Bell}

La toponymie de certains lieux de New-Bell tels que Marché Lagos, Ngonshùn, Nkolouloun, Nkongmondo, Nkolmintag, quartier Haoussa, NdzongMebi, etc., est évocatrice de sa pluriethnicité. Dans l'histoire politique du Cameroun, NewBell est une référence du mouvement nationaliste qui a eu lieu à la veille de l'indépendance, car il fut le fief de la mythique Union des Populations du Cameroun (U. P.C) née le 10 avril $1948^{4}$. Ce quartier riche d'histoire, tire ses origines des abus de la colonisation allemande.

La violation par l'Allemagne des clauses du traité germano-duala ${ }^{5}$ du 12 juillet 1884 a engendré les premiers actes du nationalisme camerounais. En effet, après une cohabitation relativement paisible avec les Africains, et pour des raisons

\footnotetext{
${ }^{3}$ Douala est la principale ville économique du Cameroun

${ }^{4}$ V. J. Ngoh, Cameroun 1884- 1885 Cent ans d'Histoire, Yaoundé, CEPER, 1990, p. 122.

L'UPC est le parti nationaliste camerounais qui lança ouvertement, dès sa naissance le 10 avril 1948, la réclamation de l'indépendance et de la réunification du Cameroun qui fut divisé en deux parties depuis sa conquête par les alliés après la première Guerre Mondiale ; parties respectivement administrées par les Français et les Britanniques.

${ }^{5}$ Le traité germano-duala a instauré le protectorat allemand au Cameroun.

Dans le présent travail, Douala désigne la ville, tandis que Duala renvoie aux peuples originaires de cette ville.
} 
sanitaires, les Allemands décident d'exproprier le clan Bell. Après 1900, certains Européens se sont plaints de la montée en puissance à Douala, de la malaria dont l'origine était attribuée à la proximité du quartier européen des cases duala, qualifiées de véritables foyers à moustiques ${ }^{6}$. Ceci ressort dans un rapport médical signé par le médecin allemand Kuhn. Ce dernier avait estimé que $72 \%$ d'indigènes étaient impaludés sur 1650 examinés ${ }^{7}$. Vers 1906, l'idée d'exproprier les Duala se fit plus persistante ${ }^{8}$. Les populations indigènes s'y opposèrent farouchement en raison de l'importance des terres qu'ils devaient perdre, ainsi que leur précieuse suprématie commerciale avec les populations de l'Hinterland du Cameroun. ${ }^{9}$

En 1908, l'administrateur Röhm succéda à Brauchitsch ${ }^{10}$. Il exerça au Cameroun jusqu'en 1914. C'est lui qui exécuta le plan d'expropriation des Duala et de la réinstallation des Africains au-delà d'une zone libre ou "Freie Zone » pour marquer effectivement une ségrégation physique entre les quartiers européens et les quartiers des Noirs ${ }^{11}$. En 1912, le nouveau gouverneur allemand, Ebermaier, mit en application le plan d'expropriation du plateau Bell, constitué des quartiers actuels de Bonanjo, Bonapriso, Bonadouma et Bali ${ }^{12}$. Malgré les nombreuses pétitions des Duala adressées au Reichstag (Parlement allemand), l'autorité allemande resta sourde. Les opérations visant l'extension du quartier administratif allemand commencèrent en $1913^{13}$. C'est à ce niveau que les faits inhérents à la naissance de New- Bell se dévoilent. En effet, l'initiative d'exproprier les Duala du plateau Bell a engendré le problème de leur recasement. Les Allemands conçurent l'appellation Neu Bell qui signifie " Nouveau Bell " et l'attribuèrent à cet espace qui se situait à l'Est de l'actuelle ville de Douala, endroit où ils avaient prévu de recaser les populations victimes de l'expropriation foncière.

Neu Bell ainsi né de la volonté et de la pugnacité des Allemands qui ont porté préjudice aux Duala, n'était alors qu'un espace réduit à 50 hectares dont $11 \mathrm{~km}$

\footnotetext{
${ }^{6}$ R. Gouellain, Douala, Ville et Histoire, Paris, Institut d'ethnologie, 1975, p. 125.

${ }^{7}$ ANY, APA 12062, principes- réformes du régime foncier.

${ }^{8}$ Gouellain, Douala ..., p. 130.

${ }^{9}$ Pour mieux saisir l'importance des terres pour les Africains en général et pour les populations camerounaises, dont les Duala en particulier, lire G. M. Dong Mougnol, "Migrations internes et problèmes fonciers au Cameroun : les cas de Makénéné et Mbangassina dans la région de Mbam, de 1926 à nos jours ", Thèse de Doctorat/Ph. D. en Histoire, Université de Yaoundé I, 2007.

${ }^{10}$ Brauchitsch fut le premier urbaniste de Douala. Il œuvra au Cameroun de février 1889 à septembre 1908. Sa mission consista à transformer ce qui était alors appelé village traditionnel africain en une ville moderne.

${ }^{11}$ G. Mainet, Douala, Croissance et servitudes, Paris, L'Harmattan, 1985, p.59.

${ }^{12}$ Ibid. p.60.

${ }^{13}$ Ibid.p.61.
} 
de routes, et où furent bâties 500 cases $^{14}$. Ce déménagement ne fut pas du goût des Duala qui, sous la houlette de leur chef supérieur, Rudolph Douala Manga Bell, initièrent une fronde contre les Allemands. Ils refusèrent catégoriquement de se

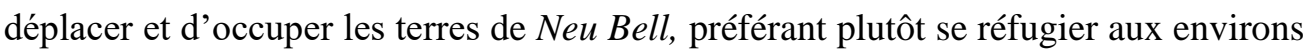
de Bali ${ }^{15}$. La répugnance que les Duala avaient à l'endroit de ce site de $\mathrm{Neu}-\mathrm{Bell}$ se traduisait par leur attachement à leurs terres ancestrales. C'est du moins ce qui fut attesté par la déclaration faite par le chef de district, Röhm, en ces termes :

Les indigènes dont les maisons ont été détruites, habitent tantôt chez d'autres indigènes dont les maisons se tiennent encore debout, tantôt vont à leurs fermes vers le Mungo comme Douala Manga qui l'a expressément déclaré ou encore restent et passent la nuit soit à la belle étoile sur leurs terrains dénudés, soit dans les cases voisines d'autres personnes ${ }^{16}$.

Pour arriver à chasser les Duala du plateau Bell, Il a fallu une action irascible des autorités allemandes qui ont fait recours aux services de la Polizeitruppe ou police allemande. Le secrétaire d'Etat aux colonies, Solf, envoya le 12 janvier 1914, 850 soldats $^{17}$ à Douala pour ébranler la conviction de ces populations. Malgré cette pression, les Duala continuèrent à dénigrer Neu Bell qu'ils qualifiaient de Mboussa Moundi ou " l'arrière-village » qui est généralement une brousse. C'était, selon eux, un lieu indigne d'un habitat pour humains ${ }^{18}$.

Le paradoxe de New- Bell devient assez perceptible à cette période allemande car, ce quartier incarnait à la fois répulsion et attraction. En effet, ce lieu si amer et invivable pour les Duala, a plutôt exercé une forte attirance sur les populations allogènes déjà relativement nombreuses à Douala, et qui éprouvaient aussi une grande difficulté à s'approprier des terres dans cette ville partiellement confisquée par le colon européen. En mars 1914, soit seulement quelques mois après sa création, Neu Bell comptait déjà 1000 habitants dont 800 étrangers d'origines variées ${ }^{19}$. Le refus des Duala d'habiter ce quartier n'avait donc pas ôté la vie à $\mathrm{Neu}$ Bell. Elle l'avait au contraire stimulée. Neu Bell, devenu plus tard New-Bell après la conquête et l'occupation de la ville de Douala par les forces alliées en 1914, s'est ainsi forgé sa réputation de quartier cosmopolite, car s'y entremêlaient, s'interpénétraient et cohabitaient des populations venues de tous les horizons.

\footnotetext{
${ }^{14}$ Ibid.

${ }^{15}$ Mainet, Douala, Croissance et servitudes..., p.61.

${ }^{16}$ A. Ruger cité par D. Abwa., Cameroun : Histoire d'un nationalisme 1884- 1961, Yaoundé, Clé, 2010, pp. 105- 106.

${ }^{17}$ D. Abwa, Cameroun : Histoire d'un nationalisme 1884- 1961, Yaoundé, Clé, 2010, p.105.

${ }^{18}$ Entretien avec J- P. Ewané Dikoumé, 76 ans, ancien combattant, Douala, 28 juin 2011.

${ }^{19}$ Mainet, Douala..., p.61.
} 
Le comportement racial allemand, qui a instauré le culte de l'exclusion de l'élément étranger, semble malheureusement avoir été récupéré plus tard par les Duala. Ces derniers ont en effet à leur tour, entretenu un repli identitaire qui s'est manifesté par le regard condescendant qu'ils portèrent sur les immigrés installés à New-Bell. Pour les Duala en effet, les New-Bellois n'étaient que des personnes inférieures qui s'étaient contentées de ce lieu dénigrant, insultant, répugnant. Habités par un complexe de supériorité, les Duala considéraient les habitants de New- Bell comme des êtres qui étaient à l'image du lieu où ils vivaient. La ségrégation ne fut donc plus seulement raciale, mais aussi intra africaine. Les Duala semblent avoir hérité du regard altier des Européens en considérant les populations new-belloises comme des parias indignes des quartiers évolués. New-Bell est dès lors devenu un véritable ghetto qui conserve une certaine originalité. En réalité, ce quartier à part dans la ville de Douala, a attiré et continue d'attirer des populations telles que les Haoussa, les Béti, les Bamiléké, les Bassa qui, au fur du temps, se sont multipliées. Elles ont partagé un même passé et vivent une même actualité. Ces populations ont souvent su manipuler leurs différences, leurs identités quant il le faut ou faire émerger leur destin commun en cas de nécessité. Il convient dès lors de saisir les migrations et installations des pionniers de New- Bell.

\section{B- La construction de la multiethnicité de New- Bell 1 -Les Bamiléké de New- Bell}

À la période précoloniale et du fait des activités des esclavagistes, quelques Bamiléké venus de l'Ouest du pays, se trouvaient déjà à la côte du Cameroun. Les Duala les appelaient Bakomkom (Mukomkom au pluriel), terme qui voulait dire esclaves bamilékée ${ }^{20}$. Ils vivaient généralement loin de leurs maîtres appélés Wenji ${ }^{21}$. L'union entre un esclave et une fille duala donnait un hybride que l'on appelait en langue Douala Muyabedi ${ }^{22}$. L'histoire retient que Georges Nkwé, qui fut l'un des premiers élèves de l'école ouverte par le missionnaire anglais Alfred Saker en 1845 à Douala, était un esclave capturé en zone bamiléké, mais qui a été baptisé du nom de Mandesi Bell, il était originaire de Bayangam ${ }^{23}$ dans l'actuel département du Koung- Khi à l'Ouest- Cameroun. De nombreux autres Bamiléké se sont retrouvés dans la ville de Douala suite aux travaux forcés entrepris pendant la période

\footnotetext{
${ }^{20} \mathrm{R}$. A Austen, Slavery among coastal middlemen, the Douala of Cameroon, Winsconsin, University of Winsconsin Press, 1977, p.308.

${ }^{21}$ Ibid.

${ }^{22}$ Ibid. p. 309.

${ }^{23}$ Slageren cité par Fabien Tiagué, "Les allogènes Bamiléké dans la ville de Douala des origines à 1955 ", mémoire de DIPES II en Histoire, ENS de Yaoundé, 2000, p.46.
} 
coloniale allemande. 1897 marque l'intrusion brutale des Allemands en pays Bamiléké, précisément dans le poste de Bana. ${ }^{24}$ Les chefs qui résistèrent à leur pénétration payèrent de lourds tributs, comme ce fut le cas du chef Bamena à qui avait été imposé de donner 400 régimes de plantains, 80 chèvres, 300 bœufs et 700 porteurs ${ }^{25}$ dont certains prirent la destination de Douala.

La crise mondiale de 1929 eut des conséquences non négligeables sur l'économie camerounaise, à l'instar de la chute du prix du cacao et du café. ${ }^{26} \mathrm{Par}$ conséquent, de nombreux agriculteurs bamiléké habitant la plaine du Moungo, migrèrent vers Douala à la recherche du bien-être. En dehors des quartiers Bépanda et Deido où ils essaimèrent en masse, New- Bell fut l'endroit par excellence où ils s'implantèrent. Durant la décennie 1930, on trouvait déjà les foyers bamiléké dans ce quartier ${ }^{27}$. Ils se regroupaient entre eux, suivant leur chefferie d'origine, formant ainsi des sous-quartiers tels que les sous-quartiers dits Bandjoun, Bangangté, etc. ${ }^{28}$

Si la présence des Bamiléké à New- Bell est ainsi attestée, il convient de signaler que l'accélération de leur émigration et leur attachement à ce quartier se fit aussi ressentir à partir de 1955. Le malaise psycho-sociologique, séquelle de la période trouble pré et post- indépendance, avait traumatisé la conscience collective bamiléké. L'exhibition des têtes coupées, les corps sans vie, déchiquetés, abandonnés et en état de putréfaction sur les sentiers de brousse et certains espaces publics mais aussi les maisons détruites et chefferies brûlées avaient créé une véritable psychose au sein des rescapés de ces événements sanglants ${ }^{29}$. D'où leur migration et regroupement identitaire dans certains sites de New- Bell. Parmi les Bamiléké qui arrivèrent à New-Bell à cette époque, l'on retient les noms de Dagobert Fampou ${ }^{30}$, Joseph Toko qui fut plus tard chef du groupement Bandjoun entre 1945 et 1960, Paul Kuété qui a été le premier chef supérieur des Bamiléké de New- Bell, Mathieu Tchamou, David Djamen, Joseph Njeya qui fut chef du groupement Ndé entre 1944 et 1962 à New- Bell , Clément Kuidjang qui a été chef du groupement Bazou de New-Bell entre 1945 et 1950 et Alfred Kemajou qui est son successeur en poste depuis $1950^{31}$.

\footnotetext{
${ }^{24}$ Tiagué, " Les allogènes Bamiléké"..., pp. 10- 11.

25 Ibid. p. 13.

${ }^{26}$ Lire l'ouvrage de Mainet, Douala..., 1985.

${ }^{27}$ Ibid.

${ }^{28}$ Mainet, Douala..., 1985. p.85.

${ }^{29}$ Cf. W. D., Foga Konefon, " La contribution des élites du Ndé au progrès du département : 1973- 2007", Mémoire de Maîtrise en Histoire, Université de Yaoundé I, 2008, pp. 39-40.

${ }^{30} \mathrm{Ce}$ dernier a plus tard dirigé la mairie de Douala IIème entre 1989 et 1994.

${ }^{31}$ Entretien avec A.Kemajou, 85 ans, chef de bloc de Nkolouloun - km5, Douala, 12 septembre 2011.
} 
L'appropriation de la majorité des terres de New-Bell par les Bamiléké, au détriment des Béti est aussi justifiée par le fait que les Béti, dont la majorité s'était enrôlée dans des activités rémunératrices tels que gardiens, employés de bureau, fonctionnaires, cheminots, optaient très souvent, une fois l'heure de la retraite sonnée, pour un retour dans leur village natal ${ }^{32}$. À leur départ, ils vendaient leurs maisons et terrains aux Bamiléké qui, eux par contre, se les léguaient de père en fils $^{33}$. Ceci a enraciné la présence des Bamiléké à New-Bell.

\section{2- Les Béti de New- Bell}

La date de l'installation des Béti à New-Bell reste confuse. Elle diverge suivant les versions collectées. La première stipule que leur présence à Douala daterait de la période coloniale. Ces derniers venaient déjà y vendre leurs produits. Voici la version recueillie auprès des Béti :

D'après nos grands-parents, les premiers Béti à arriver dans la ville de Douala furent ceux qui vinrent faire le commerce, vendre les noisettes ou les noix de palmes, les récoltes agricoles dans les comptoirs coloniaux, en échanges des vêtements, des armes, etc. La deuxième vague des Béti est arrivée à Douala à cause des travaux forcés ${ }^{34}$.

L'on s'aperçoit que les échanges commerciaux ont joué un rôle prépondérant dans l'histoire des migrations béti vers la côte du Wouri durant la période coloniale. L'ouverture vers l'hinterland voulue par les Allemands, a permit aux populations longtemps restées à l'intérieur du territoire, de s'ouvrir aux commerçants européens demeurés jusque-là à la région côtière. Elle offrit alors l'occasion aux Béti de se déplacer librement vers la côte pour la première fois ${ }^{35}$. Pour commercer avec les Blancs, ils passaient généralement par les médiateurs duala ${ }^{36}$.

L'avènement de la colonisation allemande a ouvert la voie à l'exploitation économique du territoire. Pour arriver à cette fin, le colonisateur avait besoin des porteurs ou d'une main- d'œuvre dynamique. L'échec des résistances telles que

\footnotetext{
${ }^{32}$ Mainet, Douala ..., p.361.

${ }^{33}$ Entretien avec L.Ondoa, 71 ans environ, chef de Bloc de Mbamewondo Source, Douala, 10 septembre 2011 et avec J. Ebendié, 68 ans, chef de Bloc 3 de New- Bell Kassalafam, Douala, 10 septembre 2011.

${ }^{34}$ Entretien avec D. Belibi Ndzana, 51 ans, chef supérieur des Béti de la région du Littoral, Douala 22 juin 2011.

${ }^{35}$ F. Quinn., " Rencontres dans la forêt dense : Les Béti et les Allemands, 1887-1916" in M. Z. Njeuma, Histoire du Cameroun (XIXes. - début XXes.), Paris, L' Harmattan, 1989, pp. 135- 158.

${ }^{36}$ Ibid. pp. 135- 158.
} 
celles des Mbidambani et des Bené ${ }^{37}$ ont donné l'opportunité aux Allemands de réquisitionner abondamment la dite main-d'œuvre au sein de ces populations béti vaincues. De nombreux Béti furent ainsi déportés vers la côte et affectés à la construction des infrastructures dans les villes de Buéa, Edéa et Douala ${ }^{38}$.

La toponymie de certains lieux de New-Bell est un élément important qui atteste de la relative ancienneté des Béti dans ce quartier. En effet, déjà présents sur la côte pour les transactions commerciales et les travaux forcés, les Béti ont subi l'expropriation des Duala par les Allemands. Partis avec la vague des déguerpis, ces Béti se sont réfugiés dans un lieu qu'ils nommèrent Nkongmondock c'est-à-dire en béti, "rue des manguiers" ${ }^{39}$. Après la défaite des Allemands lors de la Première Guerre mondiale, Douala tombe sous le contrôle des Français qui poursuivent le processus de son urbanisation. Le refoulement des indigènes du centre-ville et de ses environs poussa les Béti à aller s'établir dans un nouveau site qu'ils ont nommé Mbam-éwondo, situé vers l'actuel cimetière de New-Bell qu'ils disent avoir créé afin d'y enterrer leurs morts. ${ }^{40}$

$\mathrm{Au}$ moment où l'administration française envisagea la construction de la prison et le bâtiment abritant l'actuel sous-préfecture du deuxième arrondissement de Douala à New- Bell, elle dut exproprier les allogènes déjà installés sur ce site. Tout à côté, il y avait un cabanon, un lieu où l'on gardait les aliénés mentaux dont les excréments étaient déversés sur la route proche du bâtiment. Comme les agriculteurs béti de New-Bell empruntaient fréquemment ce chemin pour se rendre dans leurs plantations, ils attribuèrent à ce sentier le nom de Ndzong-Mebi, entendez « la route des excréments ». Derrière ce cabanon se trouvait alors une forêt où les Béti pratiquaient leur agriculture ${ }^{41}$. Ils s'y installèrent après leur expropriation et vulgarisèrent le nom Ndzong-Mebi qui désigna désormais cette nouvelle zone d'habitation.

\footnotetext{
${ }^{37}$ Les Mbidambani et les Bené sont des composantes du groupe béti de l'actuelle région du Centre.

${ }^{38}$ Entretien avec J- D. Anaba Etemé, 70 ans, ex- gardien de prison, Douala, 22 juin 2011.

${ }^{39}$ Entretien avec D. Belibi Ndzana, 22 juin 2011

Ce terme est aussi utilisé en Bassa et signifie nouveau village

${ }^{40}$ Entretien avec D. Belibi Ndzana, 22 juin 2011.

Mbam-éwondo voudrait dire en béti la contrée des Ewondo.

Selon la version courante au sein de la communauté béti de Douala recueillie auprès de l'informateur ci-dessus, c'est un certain Essomba Essola, célibataire sans enfant, mais acquéreur de nombreuses terres, qui aurait instruit aux siens de l'enterrer à cet endroit qui est devenu alors le cimetière des Béti, et plus tard celle des allogènes de Douala en général.

${ }^{41}$ Entretien avec D. Belibi Ndzana, 22 juin 2011.
} 
Les Béti ont profondément influencé la toponymie à partir de leur activité principale que fut l'agriculture ${ }^{42}$. En effet, le lieu appelé de nos jours Kassalafam ${ }^{43}$ était désignéà l'époque par l'expression Nkolmbong ou la « colline du manioc $»^{44}$. À peine installés dans cet endroit, l'administration française entreprit une fois de plus de repousser ces peuples allogènes vers la périphérie pour l'extension de la ville de Douala. C'est ainsi que les Béti se déplacèrent en abandonnant leurs travaux champêtres pour aller créer un autre sous-quartier à l'intérieur de New-Bell appelé Nkolouloun ou la « colline de la colère », ceci en raison de leur désaccord avec l'acte de l'administration ${ }^{45}$.

En réalité, les Béti avaient marre de leurs multiples expulsions. Ils cherchèrent des nouveaux espaces pour la pratique aisée de l'agriculture. C'est ainsi qu'ils trouvèrent deux autres endroits tel que Nkolmintag ou la « colline de la joie », du fait de la satisfaction dans le rendement agricole due à la fertilité des terres à cet endroit $^{46}$. Un autre site mis en exploitation par ces derniers fut le lieu actuellement appelé marché plantain, mais que les Béti appelaient autrefois Afoubialem, et où les cultures par excellence étaient la banane et le plantain ${ }^{47}$. Au moment des récoltes, ce lieu se transformait en un véritable marché de bananes et de plantains ${ }^{48}$.

La deuxième version est celle de Laurent Ondoa, corroborée par des archives privées de la paroisse "Notre dame de victoire " de Douala communément appelée "Sacré cœur ». Elle est recoupée par le récit de quelques pionniers béti attestant l'ancienneté de leur présence à New- Bell. D'après cette version, le sousquartier Mbam' Ewondo est le fief originel de l'installation des Béti à New-Bell ${ }^{49}$. L'un des tout premiers chefs béti de Douala fut Marcus Etemé, alias « Nyanga Boy », chef des groupements Funkel Ewondo et Mbam 'Ewondo. Son territoire de commandement allait de l'actuel marché des chèvres jusqu'au drain qui sépare les actuels sous-quartiers Mbam'Ewondo I et « source du quartier » (face à la nouvelle

\footnotetext{
${ }^{42}$ En réalité, les hommes ne sont guère vraiment agriculteurs : ce sont les femmes qui ont partie liée avec la terre. C'est pourquoi, les buttes, les semailles, les sarclages, les repiquages, les récoltes de tout ce qu'on met en terre sont leur activité principale. Cf. Philippe Laburthe Tolra, Les Seigneurs de la forêt. Essai sur le passé historique, l'organisation sociale et les normes éthiques des anciens Béti du Cameroun, Paris, L’Harmattan, 1981, p.281.

${ }^{43} \mathrm{Ceci}$ n'est en réalité qu'une déformation de l'expression anglaise Cassava farm ou le "champ de manioc".

${ }^{44}$ Entretien avec D. Belibi Ndzana, 22 juin 2011

${ }^{45}$ Ibid.

${ }^{46}$ Ibid

${ }^{47}$ Ibid.

${ }^{48}$ Entretien avec L.Tchantchou, 85 ans environ, ancienne commerçante, Nkolmintag, 22 juin 2011.

${ }^{49}$ Entretien avec L.Ondoa, 71 ans environ, chef de Bloc de Mbamewondo Source, Douala, 10 septembre 2011.
} 
route NdzongMebi) ${ }^{50}$. Les pionniers dans ce secteur étaient : Constantin Abanda, Tobie Mvogo, Pierre Bindzi, Protais Essomba Mbang, Laurent Ekani, Anne Zobo, etc. ${ }^{51}$ Tout Béti qui arrivait à Douala se dirigeait d'abord à Mbam' Ewondo ${ }^{52}$.

La concentration des allogènes et étrangers à New- Bell, commencée sous l'ère coloniale allemande, s'est poursuivie grâce à l'action de l'administrateur français Chazelas qui, en 1920, avait regroupé dans ce lieu tous les étrangers indigènes de la ville afin de les soustraire de l'esprit séditieux des Douala ${ }^{53}$. Dans l'optique de mieux gérer cette population, l'administration coloniale procéda à la nomination des chefs de regroupements qui avaient, sous leur responsabilité, plusieurs familles et clans, et dont la mission fut de servir de relais entre ladite administration et les populations.

Non loin de Mbam' Ewondo se trouvait le sous-quartier Bamiléké dont le chef était Simo. Dans ce lieu, était construite une chapelle de l'église catholique, juste au niveau de l'actuelle école New- Bell Bamiléké. Celle-ci accueillait tous les allogènes sans distinction ${ }^{54}$. Les fidèles bamiléké qui réclamaient la propriété de la dite chapelle, avaient la manie de remplir celle-ci tous les dimanches, empêchant ainsi aux autres fidèles venant des autres sous-quartiers de New-Bell de s'y introduire. Ces derniers se sentaient obligés de suivre les messes debout et à l'extérieur de la bâtisse ${ }^{55}$. Vexés par un tel comportement discriminatoire, Les Ewondo (un groupe béti) décidèrent de se construire une chapelle provisoire à côté de la résidence de leur chef Etemé où les prières étaient conduites par un catéchiste nommé Vincent Onana ${ }^{56}$. Autour de cette nouvelle maison de prière, se dressaient

\footnotetext{
${ }^{50}$ Document tiré des archives privées de L. Ondoa et relatif à la création de la Paroisse Notre Dame des Victoires de New- Bell.

51 Ibid.

${ }^{52}$ Entretien avec L.Ondoa, 71 ans environ, chef de Bloc de Mbamewondo Source, Douala, 10 septembre 2011.

${ }^{53}$ J. L.Dongmo cité par E. P.Ndanga. , " La prison de New- Bell à Douala (Cameroun), 1916

- 1960", Mémoire de Maîtrise en Histoire, Université de Yaoundé I, 2002, p.16.

Il convient de préciser que même durant la période française, les Duala ne s'étaient pas montrés totalement soumis à l'autorité coloniale. Ils avaient poursuivi par exemple leur opposition aux expropriations foncières que la France voulait entreprendre. Pour plus de détails à ce sujet, lire Dong Mougnol, " Migrations internes et problèmes fonciers au Cameroun ...", 2007.

${ }^{54}$ Document tiré des archives privées de L. Ondoa et relatif à la création de la Paroisse Notre Dame des Victoires de New- Bell.

55 Ibid.

${ }^{56}$ Ibid.

La résidence du chef Etemé était située au niveau de l'actuelle paroisse Notre Dame des Victoires.
} 
les cases dont les propriétaires, d'origine béti, étaient Constantin Abanda, Tobie Mvogo, Pierre Bindzi, Protais Essomba Mbang, Laurent Ekani, Anne Zobo ${ }^{57}$.

Plus tard, la communauté béti envisagea la construction d'une chapelle définitive. Elle émit la doléance auprès de Monseigneur Lemailloux. Accédant à leur demande, ce dernier choisit plutôt la cour de leur chefferie pour l'édification de ladite chapelle. Ce site ne plut cependant pas à toute la communauté. ${ }^{58} \mathrm{La}$ majorité d'entre eux, qui avait en effet accepté le déménagement, alla s'installer à Nkolmintag, tandis la minorité qui le désapprouvait, préféra se loger au lieu-dit Nkolouloun. Une troisième vague de Béti alla s'installer à Ndzong-Mebi, et enfin la dernière vague s'installa à Kassalafam. Parmi, les pionniers de ce site, on peut citer Messi Etienne, MbargaTsogo Simon, Zanga Philippe, Bodo Philippe ${ }^{59}$

Quelle que soit la version considérée pour expliquer la présence des Béti à NewBell, on peut déclarer sans ambages que ces derniers furent parmi les premiers allogènes à s'implanter dans ce quartier.

\section{3- Les Bassa de New- Bell}

D'après nos sources orales, les Bassa se retrouvaient déjà sur les rives du fleuve Wouri bien avant l'arrivée des Duala qui, en provenance du Congo, les y ont trouvés $^{60}$. De la cohabitation entre les deux peuples, sont nésquelques froissements dus au fait que les Bassa accusaient les Duala d'être à l'origine d'un encrassement du milieu. Ils décidèrent alors d'abandonner ces rives du Wouri aux Duala, préférant se replier vers l'intérieur, dans les lieux tels queNdog- Bati (aux alentours de Ndokoti, autre quartier populaire et populeux de la ville de Douala) ou dans l'actuel quartier dénommé « cité Sic » ou à Ndog-Simbi, l'une des banlieues de Douala ${ }^{61}$.

Concernant l'ancienneté de leur implantation dans les environs de NewBell, elle est attestée par la création par eux du quartier Nkongmondo voisin. Ce terme signifie en langue bassa « la nouvelle terre ». En effet, lors de l'expropriation des Duala par les Allemands, les Bassa avaient été éconduits vers cette «nouvelle terre » située aux portes de New- Bell. Ils infiltrèrent alors progressivement ce

\footnotetext{
${ }^{57}$ Ibid.

Selon notre informateur Laurent Ondoa, la parcelle de terrain qui sert actuellement de cour au collège Sacré cœur à Douala était la propriété d'Etemé.

${ }^{58}$ Document des archives de la paroisse Notre Dame des Victoires de New-Bell Ewondo.

${ }^{59}$ Entretien avec J. Ebendié, 68 ans, chef de Bloc 3 de New- Bell Kassalafam, Douala, 10 septembre 2011.

${ }^{60}$ Témoignages concordants de P. Matemb Bayan, 69 ans environ, chef Bassa de New- Bell Bassa (TSF) et Massoma Siméon, 42 ans environ, notable à la chefferie Bassa de New- Bell (TSF), Douala, 12 avril 2012.

${ }^{61}$ Ibid.
} 
quartier pour l'exécution des travaux forcés, la pratique du commerceet plus tard, la recherche de l'emploi ${ }^{62}$.Ceci a eu un effet attractif sur les autres groupes bassa qui vivaient dans la ville voisine d'Edéa, et même ceux des localités de Babimbi et Pouma. Une seconde vague partit alors de ces trois dernières localités pour s'installer directement à New-Bell ${ }^{63}$.

Quelques pionniers Bassa de New-Bell furent : Mapil, Bakongo, Mbock Nonga, Mongo Joseph ${ }^{64}$, Djomibuk, Matip Gustave, etc. ${ }^{65}$. Ils s'approprièrent l'espace foncier par simple défrichage. L'on ne nota aucun conflit, vue la vacuité des terres ${ }^{66}$. De cette conquête sont nés des sous-quartiers tels que Babylone, New-Bell Bassa $^{67}$.

Les populations de New-Bell, qui s'illustraient ainsi par leur diversité ethnique, ont côtoyé d'autres peuples dont les origines étrangères attestent du cosmopolitisme et de l'internationalisation du peuplement de ce quartier ; il s'agit particulièrement des Nigérians.

\section{4- Les Haoussa nigérians à New- Bell}

New-Bell abrite plusieurs peuples d'origine étrangère tels que les Maliens, Sénégalais, Nigériens, Togolais, Ghanéens, etc. Les plus nombreux restent cependant les Haoussa. Leur installation à New-Bell est justifiée par plusieurs raisons historiques.

Les Haoussa ont été spectateurs de l'expropriation foncière des Duala. Les sources orales révèlent qu'ils vivaient d'abord à Bali et Akwa avant leur transfert vers New- Bell ${ }^{68}$. Leur présence à Douala serait antérieure à la colonisation allemande. La plupart des Haoussa qu'on retrouve au Cameroun sont originaires du Nigéria. ${ }^{69}$ Leur goût très tôt prononcé pour le commerce les a amenés à entrer en contact avec le Nord- Cameroun précolonial. Ils parcouraient des longues distances pour venir vendre des kolas, des tissus, etc., dans les marchés des lamidats de

\footnotetext{
${ }^{62}$ Entretien avec M. Yebga, 82 ans, fonctionnaire retraité, New- Bell Bassa (TSF), Douala ,13avril 2012.

${ }^{63}$ Ibid.

${ }^{64}$ Aujourd'hui, il existe un lycée à New-Bell qui porte le nom de ce dernier.

${ }^{65}$ Entretien avec P. Matemb Bayan, 69 ans environ, chef Bassa de New- Bell Bassa (TSF), Douala, 12 avril 2012.

${ }^{66}$ Ibid.

${ }^{67}$ Ibid.

${ }^{68}$ Entretien avec Aladji Mériga, 76 ans, griot et premier conseiller à la chefferie supérieure des Haoussa du Littoral, Douala, 21 juin 2011.

${ }^{69}$ Lire J. L. Mengueme , " Les Haoussa de Yaoundé, des origines à 1960, allogènes ou autochtones" , Mémoire de DIPES II en Histoire, ENS de Yaoundé, 1998.
} 
l'Adamaoua. Ces commerçants ambulants ont pris le chemin du Sud- Cameroun et sont parvenus à Douala en passant par Banyo, Tibati, Foumban, puis à travers les régions des Grassfields et le Sud-Ouest du Cameroun actuel avant de s'installer à Bonabéri. ${ }^{70}$ Ils furent stoppés à ce niveau par l'absence d'un pont reliant Bonabéri à l'autre rive du fleuve Wouri. ${ }^{71}$ Leur ancienneté dans la ville de Douala est aussi signalée dès 1875 par le pasteur George Grenfell. Ce dernier, remontant le fleuve Wouri, a rencontré des tribus indigènes de l'intérieur du Cameroun, qui étaient déjà en contact avec les marchands haoussa et foulbé du Nord- Cameroun ${ }^{72}$.

Les Haoussa firent partie de la polizeitruppe ou Police allemande. Cependant, parce que mal payés, mal nourris et humiliés, beaucoup d'entre eux désertèrent pour se convertir au commerce ${ }^{73}$. Se référant aux informations livrées par Gouellain qui affirme que la polizeitruppe fut créée le 16 novembre 1891 et était composée des Hausa, de "Kruboys" et de Dahoméens ${ }^{74}$, on peut déduire qu'effectivement, les Haoussa étaient déjà présents à Douala bien avant l'expropriation des autochtones. Si l'on se fie également aux informations tirées des archives de la chefferie haoussa de Douala, il y est clairement dit que lorsque les forces alliées libérèrent le Kamerun de la possession allemande, le commandant en chef des Anglais qui débarqua à Douala le 28 septembre 1914, fut salué en libérateur par les Duala et les Haoussa venus en masse, parés de leurs habits de fêtes ${ }^{75}$.

Le transfert des Haoussa à New- Bell daterait probablement de 1913, dans la mouvance des expropriations, par les Allemands, des terres des Duala. Parmi les pionniers haoussa dans le quartier New-Bell, la mémoire collective retient les noms de Aladji Meidalailu et Aladji Garba qui étaient arrivés en 1913 et avaient trouvé une vingtaine de maisons déjà construites en feuilles séchées et les débris de

\footnotetext{
${ }^{70}$ Entretien avec Aladji Mériga, 21 juin 2011.

Et pour amples informations, lire aussi O. Bakari, " Le rôle de la communauté nigériane dans l'évolution économique et sociale de la ville de Douala de 1933 à nos jours", Mémoire de Master en Histoire, Université de Douala, 2010, pp. 11-13.

${ }^{71}$ Ibid.

${ }^{72} \mathrm{H}$. Johnston, La biographie de Grenfell, cité dans un document tiré des Archives de la chefferie traditionnelle des Haoussa de Douala, relatif à la commémoration du 25è anniversaire de l'accession au trône de sa majesté Housseini Adamou Labbo,du 16 au 18 octobre 2009, p.2.

${ }^{73}$ Document des Archives de la chefferie traditionnelle des Haoussa de Douala, relatif à la commémoration du 25è anniversaire de l'accession au trône de sa majesté Housseini Adamou Labbo, du 16 au 18 octobre 2009.

${ }^{74}$ Gouellain, Douala... , p. 128.

${ }^{75}$ Document des Archives de la chefferie traditionnelle des Haoussa de Douala, relatif à la commémoration du 25è anniversaire de l'accession au trône de sa majesté Housseini Adamou Labbo,du 16 au 18 octobre 2009, p.2.
} 
matériaux de constructions. ${ }^{76}$ Le nombre important des Haoussa de Duala a obligé l'administration coloniale française à créer une chefferie traditionnelle des Haoussa qui devint fonctionnelle en 1932- 1933 ; son premier chef était Aladji Charoko ${ }^{77}$. Certains descendants de ces pionniers nigérians ont marqué l'histoire de New- Bell, en l'occurrence Tanko Hassan, Mallam Adamou, Adamou Labo, Baba Lola Adededji, etc ${ }^{78}$. Une nouvelle vague importante de Nigérians est arrivée à New-Bell à partir de 1967, à la suite du déclenchement de la guerre civile du Biafra au Nigéria. Ces derniers migrants furent accueillis par leurs compatriotes déjà confortablement installés dans ce quartier ${ }^{79}$.

Cette réputation de New-Bell comme une terre d'accueil, lieu d'intégration multiethnique et internationale, a forgé dans ce quartier un climat fait d'une alternance permanente de rejets et de rapprochements intercommunautaires. Cette réalité propre à New-Bell, a été assez expressive lors des grands événements politiques qu'a traversés le Cameroun jusqu'en 1990.

\section{II- L'opposition politico-ethnique à New-Bell : de la veille de l'indépendance à 1982}

Les faits relatés sous ce titre s'étendent de 1955, date du bannissement de l'UPC par décret du gouvernement français du 13 juillet de cette même année, acte ayant occasionné la lutte armée pour l'indépendance du Cameroun, jusqu'en 1982, date du départ du président Ahmadou Ahidjo de la présidence de la république du Cameroun. Ces événements majeurs dans l'histoire politique du Cameroun, ont été particulièrement vécus à New-Bell en raison de la présence dans ce quartier, des ressortissants des grands groupes ethniques qui se sont illustrés comme acteurs alliés ou opposés de quelques événements ci-après décrits. New- Bell a su manipuler sa richesse ethnique en l'exhibant, de façon alternative, comme élément d'une réussite d'intégration politique, ou alors comme un véritable épouvantail, source des exclusions identitaires. Toutefois, au-delà des frasques qu'à pu vivre ce quartier, il doit sa force à sa capacité de toujours surpasser ces événements qui ont souvent dangereusement menacé sa stabilité.

\footnotetext{
${ }^{76}$ Entretien avec Aladji Mériga, 21 juin 2011.

${ }^{77}$ Document tiré des Archives de la chefferie traditionnelle des Haoussa de Douala, relatif à la commémoration du 25è anniversaire de l'accession au trône de sa majesté Housseini Adamou Labbo, du 16 au 18 octobre 2009.

${ }^{78}$ Ibid. pp. 32-35.

${ }^{79}$ Lire W. D. Foga Konefon, " Le Cameroun et la question de l'immigration nigériane : 1963-2008”, Mémoire de Master en Histoire, Université de Yaoundé I, 2010.
} 


\section{A- Le positionnement des groupes ethniques par rapport à l'interdiction de l'UPC}

L'interdiction de l'UPC avait envenimé les relations entre les populations de New- Bell. Les Bamiléké et Bassa étaient majoritairement partisans de ce parti politique qui était dirigé par Ruben Um Nyobe, un Bassa. Les Béti par contre militaient majoritairement au sein du Bloc Démocratique Camerounais (BDC) dont l'un des leaders charismatiques fut André Marie Mbida, un Béti-Eton, originaire de l'actuel département de la Lékié dans la région du Centre. Les Haoussa quant à eux, qui se sont toujours assimilés à la population peule très islamisées du NordCameroun, et de laquelle était issu Ahmadou Ahidjo, se concentraient plus dans l'Union Camerounaise (U.C), groupe politique dirigé par ce dernier ${ }^{80}$. Les adhésions politiques épousaient ainsi la coloration ethnique ; ce qui laissait présager des confrontations politico-ethniques.

Les Bamiléké et leurs alliés bassa accusaient les autres ethnies d'être de connivence avec la France qui réprimait sauvagement la rébellion dans leurs villages respectifs. D'après Matemb ${ }^{81}$, les Bamiléké et les Bassa à New- Bell s'attaquaient à ceux qui refusaient d'adhérer à la lutte pour l'indépendance du pays ${ }^{82}$. Dans cette chasse aux «traîtres », les Béti et Haoussa furent principalement indexés ${ }^{83}$. La position anti-upéciste clairement déclarée du Premier ministre d'origine Béti, AndréMarie Mbida (mai 1957-février 1958), a plus tard accentué la haine entre BassaBamiléké et Béti à New-Bell ${ }^{84}$. Avant même l'interdiction de l'UPC, durant le mois de mai 1955, des violences interethniques avaient déjà eu lieu à New- Bell et dans d'autres quartiers populaires de la ville de Douala. Elles étaient la conséquence du fameux discours prononcé par le ministre français d'outre- mer, Pierre- Henri Teitgen, le 15 mai 1955, lors de l'inauguration du pont de Wouri. Ce dernier avait invectivé les upécistes en mettant en garde tous les Camerounais contre «Félix Moumié, Ruben Um Nyobe et leurs acolytes, qui précipitent dans l'anarchie un territoire en plein essor ${ }^{85}$. En réaction à ces propos antipathiques, les upécistes ont appelé au boycott des cérémonies en l'honneur du ministre. Au moment où le défilé débuta sur le pont, Félix Roland Moumié et Ernest Ouandié tinrent un contre-

\footnotetext{
${ }^{80}$ Entretien avec Aladji Mériga, 21 juin 2011.

${ }^{81}$ Entretien avec P. Matemb Bayan, 12 avril 2012.

${ }^{82}$ Ibid.

${ }^{83}$ Témoignages concordants de P. Matemb Bayan et S. Massoma, 42 ans environ, notable à la chefferie Bassa de New- Bell (TSF), Douala, 12 avril 2012.

${ }^{84}$ Lire D.Abwa, André - Marie Mbida premier premier ministre camerounais (1917- 1980), Paris, L’ Harmattan, 1993.

85 T. Deltombe et als, Kamerun! Une guerre cachée aux origines de la Françafrique 1948

- 1971, Paris, la Découverte, 2010, pp. 168- 169.
} 
meeting devant 1500 sympathisants de l'UPC. Ce jour- là, Ernest Ouandié a lancé à la foule que: «si les policiers viennent troubler cette réunion, nous leur réservons un accueil qu'ils n'oublieront jamais $»^{86}$.

Le paroxysme de la violence est atteintà partir du 22 mai 1955. Des antiupécistes béti et haoussa, en réunion dans le quartier New- Bell,furent attaqués par les militants de l'UPC d'origine bamiléké et bassa ${ }^{87}$. Craignant que l'administration française ne détruise leur siège, les upécistes mirent sur pied un dispositif défensif, constitués de guetteurs, combattants, lanceurs de pierres, etc. ${ }^{88}$ Leur mot de passe en cas de danger était : «country na bad ${ }^{89}$. Ce jour-là, un groupe d'upécistes était venu interrompre le meeting fondateur du Front National dont l'objectif visé, avec la bénédiction du haut-commissaire français Roland Pré, était de fédérer les partis favorables à l'administration française sous la bannière d'un « nationalisme » bien tempéré ${ }^{90}$. Le fait que ce parti ait décidé d'organiser son congrès fondateur à NewBell, principal fief des nationalistes de Douala, avait hautement contribué à l'enragement des upécistes. Très tactique, l'administration coloniale avait en plus réussi à y enrôler d'anciens upécistes retournés ${ }^{91}$.Le 24 mai, l'administration coloniale mesura l'ampleur des affrontements politiques à caractère ethnique. Elle décida de protéger les points névralgiques de New- Bell en utilisant des grenades lacrymogènes. ${ }^{92}$ Malgré cela, les assaillants étaient plus armés au détriment des forces de l'ordre qui éprouvaient toujours des difficultés à les maitriser.

Ce même jour, le haut-commissaire Roland Pré se rendit à Douala et décida d'y convoyer des renforts pour pacifier le quartier des insurgés ${ }^{93}$. Le jour suivant, les upécistes défilèrent devant le secteur administratif en brandissant leurs armes. Au cours de ce même jour, des Européens furent tués, la prison de New- Bell fut assaillie, les gardes gendarmes tirèrent sur la population. ${ }^{94} \mathrm{~L}$ 'administration envoya d'autres renforts sur les lieux. La fusillade dura plusieurs heures, jusqu' à la tombée de la nuit. Lesestimations de la gendarmerie donnèrent un bilan de sept morts et une soixantaine de blessés parmi les manifestants ${ }^{95}$. Le 26 mai, de nombreuses arrestations furent opérées à New- Bell et le 27 mai, le siège de l'U.P.C de New-

\footnotetext{
86 Ibid.

${ }^{87}$ R. Gouellain, Douala, Ville et Histoire, Paris, Institut d'ethnologie, 1975, p. 334.

${ }^{88}$ Gouellain, Douala, Ville... , p.334.

${ }^{89}$ Entretien avec P. Matemb Bayan, 12 avril 2012. Cette expression voudrait dire : « le pays est menacé ».

${ }^{90}$ Deltombe et als, Kamerun! Une guerre cachée...,p.169.

${ }^{91}$ Ibid.

${ }^{92}$ Gouellain, Douala..., p. 334.

${ }^{93}$ Ibid.

${ }^{94}$ Ibid. pp.334- 335.

${ }^{95}$ Deltombe et als, Kamerun! Une guerre cachée...,p.171.
} 
Bell prenait feu, ainsi qu'une partie de ce quartier ${ }^{96}$. Ceci rendit l'atmosphère explosive entre Bamiléké-Bassa et Béti-Haoussa de New- Bell. De nombreuses rancunes furent accumulées et constituèrent des haines meurtrières. En fait, les upécistes avaient tué un Béti lors de ces échauffourées, ce qui avait amené ces derniers à créer un comité d'auto-défense contre les Bamiléké-Bassa. ${ }^{97}$ Les Haoussa, qui vivaient aussi dans la terreur, en firent autant. Ces groupes d'auto-défense ont prévalu jusqu' au lendemain de l'indépendance du territoire sous-tutelle française. L'un des témoins de cette situation révèle que

Quand Ahmadou Ahidjo devint Premier ministre le 18 février 1958 et plus tard élu Président du Cameroun en mars 1960, les Bamiléké et les Bassa, partisans de l'UPC, s'insurgèrent contre lui et nous (Haoussa) qui lui apportions notre indéfectible soutien. En 1958, Les upécistes envoyèrent une lettre à la chefferie haoussa pour signifier à leur chef supérieur qu'il serait tué si les Haoussa ne changeaient pas de camp. Cette nuit là- même, les upécistes attaquèrent la chefferie haoussa. Il y eut de nombreux blessés des deux côtés et plus de 20 personnes du côté des forces armées françaises qui protégeaient les alentours de la chefferie. Une élite haoussa du nom de Yaga, eut l'oreille coupée. Le lendemain, les Haoussa attaquèrent aussi le sous-quartier Bamiléké de New- Bell avec des machettes, couteaux, etc. Et chaque jour, entre 17 et 18 heures à l'esplanade de la chefferie haoussa, des séances d'entrainement aux tirs d'arcs et flèches, de lance et pierres furent organisées dans l'intention d'intimider les Bamiléké upécistes. La promptitude avec laquelle ces derniers avaient attaqué les Bamiléké a fait que ces derniers nous appellent les sanguinaires, les vrais maquisards ${ }^{98}$.

La violence politique à caractère ethnique dans New- Bell, s'illustra aussi à travers un fait qui s'est produit le 21 septembre 1959. Ce jour, Mbassi Bouchar, chef du groupement des Eton-Est, fut tué à la suite des coups de machettes des « maquisards $»^{99}$. En représailles, Paul Kuété, chef du groupement bamiléké, fut blessé par des assaillants dans le sous-quartier dit Kassalafam ${ }^{100}$. Le 9 mai 1960 vers 19 heures, Mathieu Tchamou fut tué à coups de pistolet à Kassalafam ${ }^{101}$. En avril 1960, Bangoulap, le chef Bamiléké du sous- quartier Nkolmintag et Joseph Toko,

\footnotetext{
${ }^{96}$ Gouellain, Douala, ville et Histoire..., pp. 334-335.

${ }^{97}$ Entretien avec D. Belibi Dzana, 22 juin 2011.

${ }^{98}$ Entretien avec Aladji Mériga, 76 ans, 21 juin 2011.

${ }^{99}$ F. X. Ngomsi , " De la guérilla urbaine à Douala : 1955- 1964", Mémoire de Maîtrise en Histoire, Université de Yaoundé I, 2005, p. 82.

«Maquisard » était l'expression couramment utilisée pour désigner les combattants de l'UPC.

${ }^{100}$ Ngomsi , " De la guérilla urbaine à Douala ...", p.82.

${ }^{101}$ Ibid.
} 
chef du groupement Bandjoun, étaient exécutés à coup de pistolets et de machettes ${ }^{102}$.

Ces événements sanglants perpétrés à New- Bell par des communautés animés de l'esprit de vengeance, se poursuivirent avec l'exécution d'un Béti nommé Atéba ${ }^{103}$. L'instabilité politico-ethnique de New-Bell a favorisé une étroite collaboration entre Jean Fochivé, responsable de la sureté nationale et Joseph Bélibi, chef des Béti de Douala. Ceci a renforcé l'action des comités de défense béti contre les insurgés upécistes ${ }^{104}$. La langue utilisée au sein de ces comités fut d'ailleurs le béti, ce qui a favorisé l'interpellation de tout inconnu passant dans le secteur sous contrôle des Béti ${ }^{105}$. La stigmatisation ethnico-politique s'enracinait ainsi à New-Bell. Aucune stabilité ne semblait plus solide. Même les alliances circonstancielles volèrent parfois en éclats, sous la pression de l'évolution de l'actualité politique.

\section{B - Le vacillement de l'alliance Béti-Haoussa de New- Bell}

Jusque-là sans encoche, les rapports entre Béti et Haoussa de New-Bell ont pris une tournure conflictuelle au lendemain de la démission d'André-Marie Mbida de ses fonctions de premier-ministre, et surtout son remplacement par Ahmadou Ahidjo, un Peul musulman, allié des Haoussa. L'opposition qui s'en est suivie entre Ahidjo et Mbida a eu des graves répercussions à New-Bell. Elle a amené les Haoussa à considérer les Béti et leur leader Mbida, comme nuisibles à l'épanouissement politique de leur proche qu'était Ahidjo. Dès lors, un climat de suspicion réciproque s'empara des deux communautés. La fracture entre Haoussa et Béti fut perceptible dès le retour au Cameroun de Mbida, le 4 mars 1959, après treize mois d'exil à Conakry. Les Béti de Douala en général, et ceux de New-Bell en particulier, lui réservèrent un accueil chaleureux. Le nombre de personnes mobilisées à cet effet, ainsi que la consonance des patronymes respectifs des leaders de ces masses, prouvent que dans cet environnement d'opposition entre le nouveau Premier Ministre Ahidjo et son prédécesseur, les Béti de Douala s'étaient clairement positionnés en faveur de Mbida. Retenons ces détails relevés par Daniel Abwa qui affirme que

À Douala, il fut accueilli au port par son épouse et celle du député MangaBilé, par TsallaMekongo et Amougou Nguélé, députés démocrates Camerounais. A la descente du bateau, il fut acclamé par une foule évaluée à

\footnotetext{
102 Ibid.

${ }^{103}$ Entretien avec L.Ondoa, 71 ans environ, chef de Bloc de Mbamewondo Source, Douala, 10 septembre 2011.

${ }^{104}$ Entretien avec D. Belibi Dzana, 22 juin 2011.

${ }^{105}$ Entretien avec L.Ondoa, 10 septembre 2011.
} 
près de trois mille personnes et un cortège de voitures et de cyclistes l'accompagna jusqu' à Kasalafam au domicile du chef supérieur des Bétis à Douala, Belibi. A la chefferie, il fut accueilli par près de cinq mille sympathisants et après toutes les manifestations de la joie des retrouvailles, Mbida fut invité à une réception moins tumultueuse et plus intime au domicile de Noah Martin. Il s'agissait d'un conseiller municipal Béti $(\ldots)^{106}$.

Jean Paul Amougou qui nous a accordé un entretien, va même plus loin en affirmant sans ambages qu' "À son retour d'exil, après avoir été accueilli par ses pairs béti, il leur demanda clairement, par l'entremise du chef Bélibi, de continuer à le soutenir au détriment d'Ahidjo. Ceci s'était passé à la chefferie supérieure des Béti du littoral, sis à Nkolouloun ${ }^{107}$. Il convient de signaler qu'au préalable, de nombreuses autres manifestations d'hostilités d'André- Marie Mbida à l'endroit du gouvernement d'Ahidjo furent perçues par les Haoussa de New- Bell comme une provocation des Béti à leur égard. Abwa illustre cette situation par ce récit :

Au mois de mars 1958, André-Marie Mbida eut une fois de plus à manifester sa mauvaise humeur devant les honneurs rendus à Ahmadou Ahidjo. Ce dernier revenait d'un de ses multiples voyages en France et comme d'habitude, il avait été demandé à la population de Yaoundé, et notamment aux élèves, de venir rendre les honneurs au cortège du nouveau Premier ministre. Or, ces derniers attendaient le passage de ce cortège devant la demeure de l'ex-Premier ministre qui, avec l'aide de son épouse et de ses hommes, décidèrent de les chasser. Il s'en suivit une rixe au cours de laquelle il y eut quelques blessés légers qui furent conduits à l'hôpital ${ }^{108}$.

Cette attitude de Mbida à l'endroit d'Ahidjo a profondément entamé la sérénité des relations Haoussa et Beti de New-Bell. Quelques escarmouches mineures furent fréquemment notées. Ce n'est qu'avec le temps que, convaincus enfin du rapport de force qui n'était plus en leur faveur, du fait de la consolidation de l'autorité d'Ahidjo à travers tout le pays, que les Béti de New-Bell ont finalement rallié sa vision politique. Mbida tombe alors en disgrâce. Dieudonné Bélibi Ndzana, chef supérieur des Béti, fut l'un des artisans de ce nouveau rapprochement. Il avait acquis une certaine audience auprès du Président Ahmadou Ahidjo ; appelant ensuite les siens à soutenirce dernier. Son action a suscité l'adhésion massive des Béti de New-Bell à l'Union Camerounaise, groupe politique devenu parti en 1958 et dirigé par Ahmadou Ahidjo ${ }^{109}$. Pour arriver à cette nouvelle alliance entre les deux communautés, un autre personnage énigmatique devenu plus tard une figure

\footnotetext{
106 Abwa, André- Marie Mbida...,p.233.

${ }^{107}$ Entretien avecJ. P. Amougou, 70 ans, ex- membre du B. D.C, Douala, 23 juin 2011.

${ }^{108}$ Abwa, André- Marie Mbida... , p. 152.

${ }^{109}$ Entretien avec J- P. Amougou, 70 ans, ex- membre du B. D.C, Douala, 23 juin 2011.
} 
politique incontournable à New-Bell, a dû jouer un rôle important, il s'agit d'un certain Tanko Hassan.

Les échos de l'ébullition politico-ethnique de New-Bell n'avaient pas échappé à Ahmadou Ahidjo.Ce dernier était en effet conscient de la répugnance dont il était l'objet au sein des autres communautés installées à New-Bell, en l'occurrence les Bamiléké et Bassa.Il confia alors secrètement à l'Haoussa Tanko Hassan, la mission non officielle de trouver un moyen de panser les blessures psychologiques ayant affecté les Bamiléké, du fait des répressions exercées contre les upécistes de New-Bell ${ }^{110}$. C'est alors que Tanko Hassan sélectionna au sein de la communauté bamiléké, quelques membres dont entre autres Kadji Defosso, Koloko Lewis, Somhaing André, en faveur desquels le Président Ahmadou Ahidjo intervint pour l'octroi des crédits bancaires qui leur ont permis de monter des structures commerciales et industrielles, ce qui en a fait plus tard de puissants hommes d'affaires très réputés au Cameroun ${ }^{111}$. Cette magnanimité du jeune régime a eu pour effet de susciter une adhésion massive des Bamiléké à l'UC, parti dirigé par Ahmadou Ahidjo.Lequel parti est finalement devenu le creuset de l'unité intercommunautaire dans ce tumultueux quartier de Douala. Ce stratagème a fait aussi de Tanko Hassan, le seul et incontestable maître du jeu politique à New- Bell pendant les vingt-deux années qu'Ahidjo a passé à la Présidence de la République du Cameroun. ${ }^{112}$

Il convient néanmoins de signaler que l'élite bamiléké de New-Bell ne fut cependant pas entièrement neutralisée. Certains tels que Dagobert Fampou, manifestaient en permanence leur opposition farouche à l'hégémonie peul-haoussa de New-Bell. Ce dernier accusait Tanko Hassan d'avoir instrumentalisé les

\footnotetext{
${ }^{110}$ Entretien avec Aladji Mériga, 21 juin 2011.

${ }^{111} \mathrm{D}$ 'après Deltombe, les élites bamiléké furent les premières bénéficiaires de cette stratégie. Le contexte des événements se situe dans la décennie 1960, exactement au lancement du premier plan quinquennal de développement (1961 -1966) au cours duquel l'économie camerounaise prit un décollage. Le Président Ahidjo utilisa cette arme économique pour pacifier les revendications sociales dans l'ensemble du pays. En pays Bamiléké où la rébellion avait atteint son paroxysme dans les années 60 . Ahidjo accorda une grande liberté économique aux élites de l'Ouest "Bamiléké ». A la suite de leur ralliement et de leur soutien aux élections de 196, il les laissa s'enrichir en jouant sur les droits de douanes provisoires entre les deux Cameroun et sur les erreurs techniques qui furent commises. En outre, Victor Kanga, ministre des finances de l'époque, avait aménagé les programmes d'importation dans l'optique qui leur était favorable. Cette stratégie leur a permis non seulement de s'enrichir, mais aussi d'oublier leurs appétits politiques. Lire Deltombe et als, Kamerun! Une guerre cachée...,2010.

${ }^{112} \mathrm{Il}$ convient d'ailleurs de noter que c'est Tanko Hassan qui fut le président départemental de l'Union Nationale Camerounaise (UNC) dans le Wouri. L'UNC est le parti unifié né en septembre 1966 après la fusion entre l'UC et les autres partis du Cameroun occidental.
} 
Bamiléké de ce quartier et d'avoir réussi à les reléguer en arrière-plan au détriment des Haoussa. ${ }^{113}$ A titre d'illustration à cette thèse, il s'appuya sur des pratiques courantes. Le 20 mai de chaque année en effet ${ }^{114}$, Tanko Hassan demandait aux femmes bamiléké de participer massivement au défilé. Celles-ci le faisaient avec un apparent engouement, de peur d'être taxées de subversives. Par contre, les femmes ressortissantes de la communauté peule et haoussa étaient exemptées d'un tel devoir. Lors donc d'une réunion politique qui eut lieu à la permanence du parti de Bonanjo en 1963, Dagobert Fampou eut le courage de prendre la parole et d'interpeller publiquement Tanko Hassan sur cette discrimination dans le traitement des femmes de New-Bell. Avec véhémence, il dénonça ouvertement cette attitude qu'il jugeait injuste. Ces remarques embarrassèrent profondément Tanko et coûtèrent à Fampou un internement de six mois à la prison de New-Bell ${ }^{115}$.

Malgré, ces résistances individuelles, Tanko Hassan ne lésina pas sur l'appui à apporter à ceux des Bamiléké qui avaient choisi la soumission au régime d'Ahidjo, marginalisant ainsi les opposants. Les bénéficiaires en ont pris goût. En 1982, les oppositions meurtrières entre Haoussa et Bamiléké n'étaient plus qu'un lointain et amer souvenir au point où, lorsqu'Ahidjo annonça sa démission de ses fonctions de Président de la République le 04 novembre, une délégation des Bamiléké de NewBell alla rencontrer Tanko Hassan dans l'espoir d'obtenir une audience auprès du Président afin de le convaincre de revenir sur sa décision. ${ }^{116}$ Ceci est l'illustration parfaite de ce que Biyiti bi Essam décrit en affirmant que le veuvage observé chez certains hommes politiques, certains hommes d'affaires, ajoutés à celui de certaines couches et certains groupes de la population qui s'identifiaient au régime, ont aussi lourdement plaidé pour l'annulation par Ahmadou Ahidjo de sa décision de quitter le pouvoir ${ }^{117}$.

Concernant l'élite politique Bassa de New- Bell, elle eut une attitude plutôt mitigée, faite de radicalisme et de ralliement au régime d'Ahidjo. Certains, tel que Bayiha Nlep Mathieu refusèrent catégoriquement d'adhérer ni à l'UC, ni à l'UNC. D'autres par contre, à l'instar de Koum Bienvenue et Hiéga Ernest, regagnèrent les rangs du parti. Tanko Hassan en aurait d'ailleurs fait ses bras droits ${ }^{118}$.

\footnotetext{
${ }^{113}$ Entretien avec F.Nganwa, 61ans, Cheminot rétraité, Douala, 23 juin 2011.

${ }^{114} \mathrm{Le} 20$ mai est le jour de la fête nationale au Cameroun. Il commémore en effet l'unification entre Cameroun oriental francophone et le Cameroun occidental anglophone après le référendum du 20 mai 1972.

${ }^{115}$ Entretien avec A. Kemajou, 12 septembre 2011.

${ }^{116}$ Entretiens avec A. Kemajou et Aladji Mériga.

${ }_{117}$ J. P. Biyiti bi Essam, Cameroun : complots et bruits de bottes, Paris, L'Harmattan, 1984, p.49.

${ }^{118}$ Entretien avec P. Matemb Bayan, 12 avril 2012.
} 


\section{C- La résurgence des oppositions (1982-1990)}

Avec le changement de régime survenu en 1982 au Cameroun, la cohabitation politique interethnique de New- Bell fut de nouveau secouée. L'arrivée de Paul Biya, un Béti du Sud au pouvoir, suite à la démission du nordiste musulman Ahidjo, a galvanisé les Béti de New- Bell. De 1982 à 1984, les Haoussa donnèrent leur soutien total au dauphin constitutionnel d'Ahidjo, jusqu' au coup d'Etat du 06 avril 1984 perpétré par les barons de l'ancien régime, fidèles à Ahidjo et majoritairement originaires du grand-Nord ${ }^{119}$. Leur entreprise visant à renverser le nouveau Président de la République a confirmé aux yeux de tous, leur statut d'opposants politiques. Ceci a abouti à une résurgence de la haine politico-ethnique entre Béti d'une part et les Peuls et leurs assimilés haoussa d'autre part. New-Bell s'illustra de nouveau comme un terrain d'expression de ce jeu d'alternance entre conflits et apaisements que se livraient les communautés qui s'y sont établies, ce au rythme des événements politiques nationaux.

En 1984, l'arrestation suivie de l'emprisonnement de Tanko Hassan fut la goutte d'eau qui fit déborder le vase ${ }^{120}$. Toute la communauté musulmane peulhaoussa de New-Bell se sentit très choquée car un tel crime était impensable quelques années avant. La chute de Tanko sonna comme un coup de massue sur leurs têtes. La nouvelle relation tumultueuse entre Béti et Haoussa raviva l'alliance politique entre Bamiléké et Haoussa. Voulant profiter du retour d'un des leurs au pouvoir, les Béti de New-Bell s'attelèrent à renverser le cours de l'histoire dans ce quartier. C'est ainsi que lors des élections législatives de 1988 et contre toute attente, un Béti de New-Bell fut élu député à l'Assemblée Nationale, il s'appelait Grégoire Owona. Ce fut une grande première dans les annales de ce quartier ${ }^{121}$. Cette élection fut la preuve que les Haoussa et Bamiléké avaient perdu le contrôle politique dans New-Bell et que les Béti ressuscitaient politiquement.

$\mathrm{Au}$ début de la décennie 1990, face aux revendications appelant à la réinstauration du multipartisme, l'on a vu réapparaître les oppositions politicoethniques à New-Bell. En général, contrairement aux Béti qui soutenaient le régime de Paul Biya, les jeunes Bamiléké, Haoussa et Bassa sont massivement descendus dans les rues où ils manifestèrent bruyamment. Après la réinstauration de multipartisme tant réclamé en 1990, plusieurs partis politiques ont été créés. L'adhésion à ceux-ci a parfois épousé des clivages tribaux perceptibles à New-Bell.

\footnotetext{
${ }^{119}$ Le grand Nord regroupe les régions actuelles de l'Adamaoua, du Nord et de l'ExtrêmeNord du Cameroun

${ }^{120}$ Tanko Hassan fit partie des personnalités influentes de l'ancien régime qui furent arrêtées après le coup d'Etat du 6 avril 1984.

${ }^{121}$ Entretien avec D. Belibi Dzana, 22 juin 2011.
} 
Dans le Social Democratic Front (S.D.F) dirigé par John Fru Ndi ${ }^{122}$, se trouvaient majoritairement les Bamiléké et les populations anglophones originaires del'actuelle région du Nord- Ouest. L'Union Nationale pour la Démocratie et le Progrès (UNDP), d'abord dirigée par Samuel Eboua qui fut destitué au profit de Bello Bouba Maïgari ${ }^{123}$, rassemblait en majorité les Peul et Haoussa. L'Union des populations du Cameroun (U.P.C) renfermait majoritairement les Bassas et les Béti préféraient le Rassemblement Démocratique du Peuple Camerounais (RDPC). La mort du parti unique qui autrefois, avait été un élément fédérateur de la différence ethnique à New-Bell, semblait ainsi rouvrir les plaies non pansées des années de braise. L'avenir à New-Bell était donc très redouté à la fois des populations et des pouvoirs publics.

\section{Conclusion}

Lorsque les colons allemands ont créé le quartier de New-Bell au début du XXè siècle, ils avaient un souci principal, celui de recaser les populations duala qu'ils étaient obligés d'exproprier pour des besoins coloniaux d'urbanisation. Répulsif pour les Duala mais très attrayant pour les populations allogènes venues de tout bord, New-Bell s'est très vite transformé en une terre d'intégration nationale et internationale. Curieusement, ce quartier avait rassemblé majoritairement des populations qui ont eu une très grande implication dans la dynamique politique qui a défini le Cameroun de 1913 à 1990. D'événement en événement, l'on a vécu la prise de position très partisane des acteurs de la scène politique dans ce quartier. Une sorte de nombrilisme politique très marqué s'afficha à New-Bell, créant fréquemment des alliances politico-ethniques circonstancielles. Des leaders en ont émergé, qui ont su manipuler la fibre ethnique pour consolider leur assise politique. Des hommes d'Affaires sont nés, du fait de la volonté des dirigeants, d'apaiser le climat conflictuel de New-Bell. De tout temps, New-Bell a été une menace que jusqu'ici, le pouvoir a su calmer. Le paradoxe de New-Bell est que bien que n'ayant pas la capacité de dissiper ses différences ethniques, ce quartier a tout de même la force de gérer ses oppositions politico-ethniques sans jamais tomber dans le piège d'une déflagration permanente. Cependant, New-Bell demeure une poudrière que les autorités camerounaises feraient mieux de surveiller de trop près.

\footnotetext{
${ }^{122}$ John FruNdi est un ressortissant de la région du Nord-Ouest où est majoritairement parlé l'anglais comme langue officielle du Cameroun.

${ }^{123}$ Samuel Eboua fut l'un des barons du régime Ahidjo. Ancien Secrétaire Général à la Présidence de la République. Il était originaire du Moungo dans l'actuelle région du Littoral. Bello Bouba Maïgari par contre est un Peul du Nord-Cameroun.
} 


\section{Références bibliographiques}

\section{A- Ouvrages}

Abwa, D., André-Marie Mbida premier premier ministre camerounais (19171980), Paris, L' Harmattan, 1993.

.......... ., Cameroun : Histoire d'un nationalisme 1884-1961, Yaoundé, Clé, 2010.

Austen, R. A., Slavery among coastal middlemen, the Douala of Cameroon, the university of Wionsconsin press, 1977.

Biyiti bi Essam, J. P., Cameroun : complots et bruits de bottes, Paris, L' Harmattan, 1984, p.49.

Cadiou, $\mathrm{F}$ et als. , Comment se fait l'Histoire? Pratiques et enjeux, Paris, L'

Harmattan, 2003.

Deltombe, T., et als, Kamerun! Une guerre cachée aux origines de la Françafrique 1948 - 1971, Paris, la Découverte, 2010.

Gouellain, R., Douala, Ville et Histoire, Paris, Institut d'ethnologie, 1975.

KegneFodouop, Le Cameroun Autopsie d'une exception plurielle en Afrique, Paris,

L' Harmattan, 2010.

Laburthe - Tolra P., Les Seigneurs de la forêt. Essai sur le passé historique, l'organisation sociale et les normes éthiques des anciens Béti du Cameroun, Paris, L'Harmattan, 1981.

Mainet, G., Douala croissance et servitudes, Paris, L' Harmattan, 1985.

Mbock C. G. (ss la dir. de), Les conflits ethniques au Cameroun : quelles sources, quelles solutions?, Yaoundé, Edition Saagraph, 2000.

Kosseleck, R., Le futur, le passé. Contribution à la sémantique des temps historiques, Paris, Ehess, 1990.

Ngoh, V.J., Cameroun 1884- 1885 Cent ans d'Histoire, Yaoundé, CEPER, 1990.

Njeuma, M. Z.,Histoire du Cameroun (XIXe S. début XXe S.), Paris, L' Harmattan, 1989.

\section{B- Travaux académiques}

Bakary, " La force publique au Kamerun sous administration allemande : 18841916", mémoire de maîtrise en Histoire, Université de Yaoundé I, 1997.

Bakari, O.," Joseph Paraiso : premier chef supérieur des «étrangers » au Cameroun 1915- 1978 ", Mémoire de Maîtrise en Histoire, Université de Douala, 2004. 
"Le rôle de la communauté nigériane dans l'évolution économique et sociale de la ville de Douala de 1933 à nos jours", mémoire de Master en Histoire, Université de Douala, 2010.

Dong Mougnol G. M., " Migrations internes et problèmes fonciers au Cameroun : les cas de Makénéné et Mbangassina dans la région du Mbam, de1926 à nos jours ", Thèse de Doctorat/Ph. D. en Histoire, Université de Yaoundé I, 2007.

Foga Konefon, W. D., "La contribution des élites du Ndé au progrès du département : 1973- 2007", mémoire de maîtrise en Histoire, Université de Yaoundé I, 2008.

....." Le Cameroun et la question de l'immigration nigériane : 1963- 2008 ", Mémoire de Master en Histoire, Université de Yaoundé I, 2010.

Mamoudou, “ Les relations interlamidales de la fondation de l'émirat de l'Adamaoua (1809) à 2000", Thèse de Doctorat/Ph. D. en Histoire, Université de N'Gaoundéré, 2004.

Manga, T., " Le développement du catholicisme dans la ville de Yaoundé (19551998)", mémoire de maîtrise en Histoire, Université de Yaoundé I , 2005.

Ndanga E. P., “ La prison de New- Bell à Douala (Cameroun), 1916 - 1960", Mémoire de Maîtrise en Histoire, Université de Yaoundé I, 2002.

Ngomsi, F. X., " De la guérilla urbaine à Douala : 1955- 1964", Mémoire de Maîtrise en Histoire, Université de Yaoundé I, 2005.

Tiagué, F.," Les allogènes Bamiléké dans la ville de Douala des origines à 1955", Mémoire de DIPESII en Histoire, ENS de Yaoundé, 2000.

\section{C-Archives}

Document tiré des Archives de la chefferie traditionnelle des Haoussa de Douala, relatif à la commémoration du 25è anniversaire de l'accession au trône de sa majesté HousseiniAdamouLabbo, du 16 au 18 octobre 2009 ANY, APA 12062, principesréformes du régime foncier.

\section{D- Sources orales}

1- $\quad$ Aladji Mériga, 76 ans, griot et premier conseiller à la chefferie supérieure des Haoussa du Littoral, Douala, 21 juin 2011.

2- $\quad$ Anaba Etemé, J.D, 70 ans, ex- gardien de prison, Douala, 22 juin 2011.

3- Belibi Ndzana, D, 51 ans, chef supérieur des Béti de la région du Littoral, Douala 22 juin 2011.

4 Ebendié, J, 68 ans, chef de Bloc 3 de New- Bell Kassalafam, Douala, 10 septembre 2011.

5- $\quad$ EwanéDikoumé,J- P, 76 ans, ancien combattant, Douala, 28 juin 2011. 
6- $\quad$ Kemajou,A, 85 ans, chef de bloc de Nkolouloun - km5, Douala, 12 septembre 2011.

7- $\quad$ Ondoa,L, 71 ans environ, chef de Bloc de Mbamewondo Source, Douala, 10 septembre 2011.

8- Massoma Siméon, 42 ans environ, notable à la chefferie Bassa de New- Bell (TSF), Douala, 12 avril 2012.

9- MatembBayan, 69 ans environ, chef Bassa de New- Bell Bassa (TSF), 12 avril 2012.

10- Nganwa, F, 61ans, Cheminot retraité, Douala, 23 juin 2011.

11- Yebga, M, 82 ans, fonctionnaire retraité, New- Bell Bassa (TSF),

Douala ,13avril 2012.

12- Tchantchou, 85 ans environ, ancienne commerçante, Nkolmintag, 22 juin 2011. 